\title{
Detecting Discontinuities Using Volumetric Seismic Curvature Extraction: Case Study Using Deepwater Niger Delta 3D Seismic Data
}

\author{
Babangida Jibrin ${ }^{1, *}$, Isah Aliyu Goro \\ ${ }^{1}$ Department of Geology and Mining, Ibrahim Badamasi Babangida University, Nigeria \\ ${ }^{2}$ Department of Geology, Federal University of Technology, Nigeria
}

Copyright $(\mathcal{C} 2015$ by authors, all rights reserved. Authors agree that this article remains permanently open access under the terms of the Creative Commons Attribution License 4.0 International License.

\begin{abstract}
Techniques for detecting discontinuities by computing the absolute maximum curvature of seismic traces have been applied to a 3D seismic data acquired in the deep-water Niger Delta. Volumetric dip and azimuth of the seismic traces were computed from the primary seismic reflection data and structurally improved to highlight discontinuities based on the changes in dip trends that may be due to structural deformation. The dip data was used with the seismic reflection data to compute the absolute maximum curvature of the seismic traces using the concept of dip-steering. The attributes detected a WNW-ESE trending zone of dip and curvature anomalies representing discontinuities related to folding and thrusting in the area. These anomalies are hardly visible in the seismic reflection data. The workflow demonstrates the usefulness of volumetric seismic dip and curvature computation in rapid analysis of discontinuities prior to detail structural interpretation.
\end{abstract}

Keywords Offshore, Niger Delta, Seismic Data, Dip and Azimuth Volumes, Maximum Curvature, Discontinuities

\section{Introduction}

Over the years, several geometric seismic attributes have been used by seismic interpreters to delineate geological features for oil and gas exploration $[1,2,3]$. However, one of the challenges of interpreting structures in seismic data is the ability to distinguish between seismic anomalies related to real geological features and those related to noise, both of which may occur in the same data. In addition, the resolution limits of most seismic data could impact negatively on the resolvability of seismic events. The coherence cube [4] has traditionally been used to highlight faults and edges of stratigraphic features (e.g. channels) that the seismic amplitude data may not show. A recent development in seismic attribute computation is the concept of volume extraction of multi-trace seismic curvature steered by seismic dip data $[5,6]$. In the past, previous workers $[7,8]$ have computed the curvature of surfaces interpreted directly from 3D seismic data.
Although multitrace similarity attribute has previously been used to detect faults in the deep-water Niger Delta [9], in this article, we describe techniques for utilizing the absolute maximum curvature of seismic traces along structural dips for detecting discontinuities. The techniques have been applied to a seismic data acquired in water depths ranging from $\sim 1000$ to $2000 \mathrm{~m}$ in the deepwater Niger Delta (Fig. 1). Faults are important in oil and gas exploration as a conduit and/or barrier to the flow of hydrocarbon fluids [10, 11]. In recent years, considerable exploration efforts have focused on the deepwater Niger Delta with faults being the primary exploration targets. As the need for an improved understanding of sub-surface fault framework is intensified, accurate detection and mapping of structural discontinuities using specially processed seismic attributes will become even more important as an integral part of seismic interpretation workflows. We use block diagrams and timeslices extracted from the computed seismic attribute data to demonstrate that the attributes can add value to structural interpretation by providing the interpreter with a potentially powerful tool for detecting and mapping complex subsurface geological features that may not be clearly seen in standard seismic reflection data.

\section{Materials and Methods}

The post-stack time-migrated 3D data have inline and crossline spacing of 25 and $12.5 \mathrm{~m}$ respectively. The recording interval is $9 \mathrm{~s}$ with a 4 msec sampling rate. The data have been zero-phased migrated with vertical scale in seconds (sec) two-way travel time. Spectral analysis (Fig. 2) shows that the dominant frequency varies from 40 to $60 \mathrm{~Hz}$ between 3.0 and 6.0s two-way travel time. A frequency of $\sim 22 \mathrm{~Hz}$ that appears to be the strongest in the amplitude spectrum plot was used to calculate the resolution of the data. The maximum vertical resolution is $\sim 18 \mathrm{~m}$ at shallower sections but deteriorates to $>30 \mathrm{~m}$ in the deeper sections. Vertical resolution was computed using an interval velocity versus depth plot from offshore Niger Delta [12]. The workflow applied to detect discontinuities in the data is summarized in Figure 3. 


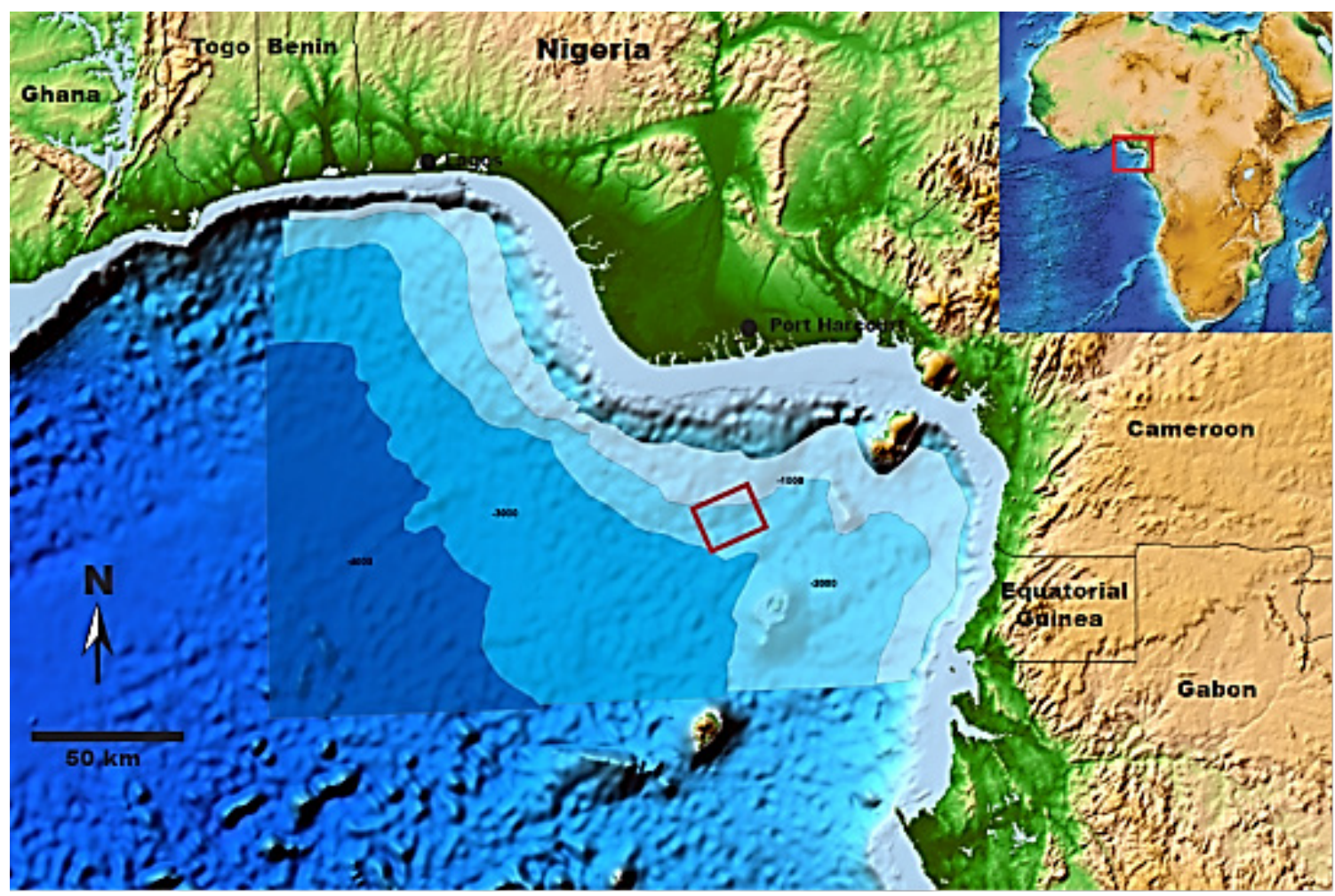

Figure 1. Shaded relief map of the Gulf of Guinea showing the study area.

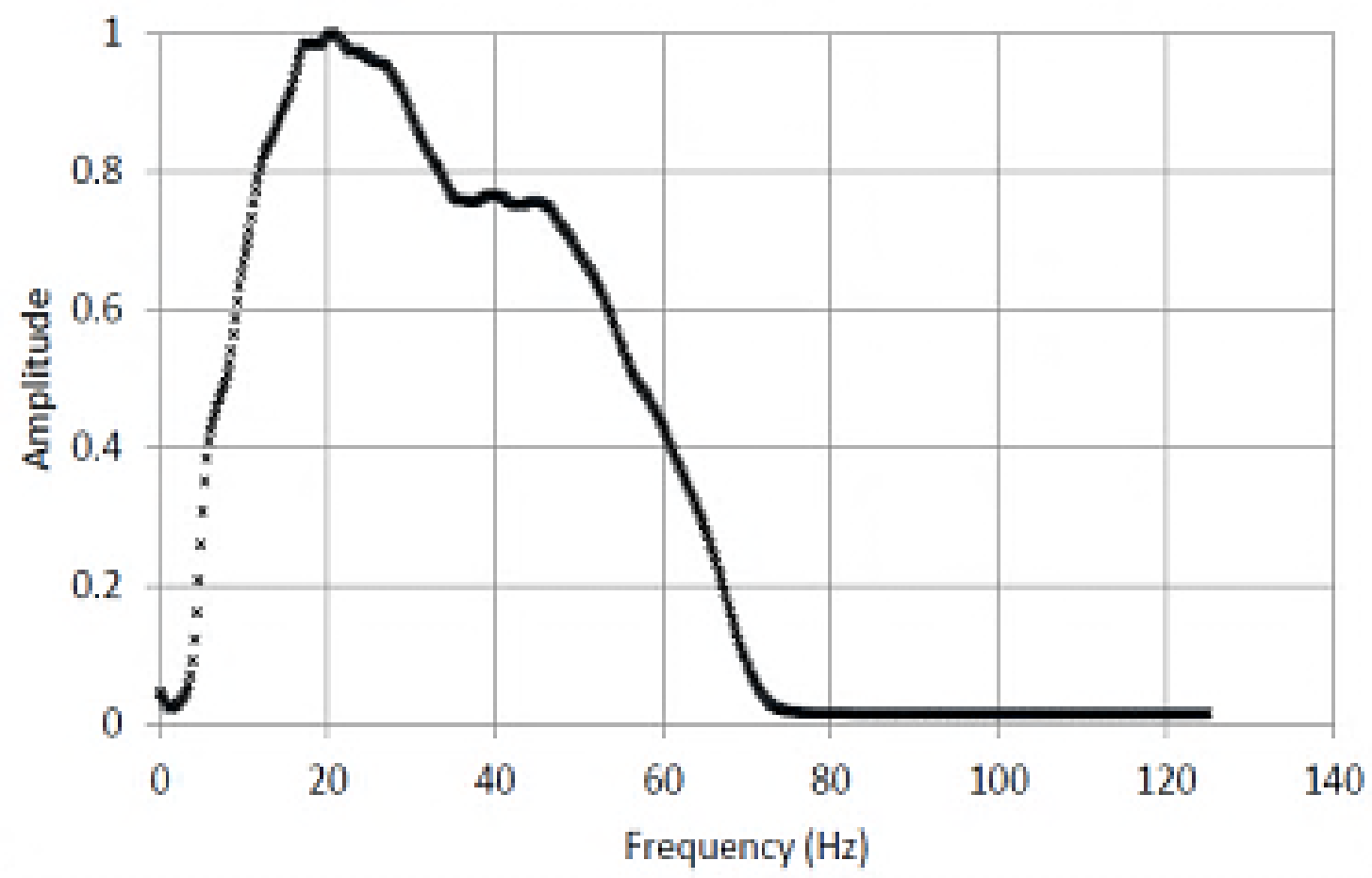

Figure 2. Frequency bandwidth extracted between 3 and $6 \mathrm{sec}$ two-way travel times. 


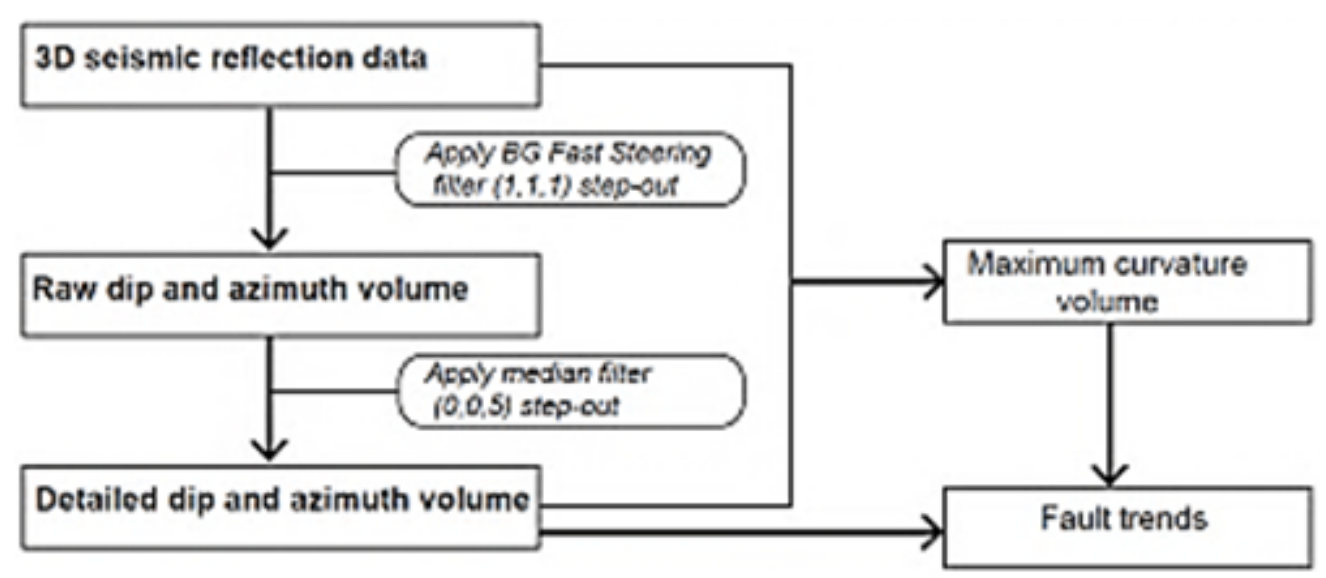

Figure 3. Workflow for detecting discontinuities applied to the data.

Table 1. Parameter setting for computing dip and azimuth volumes

\begin{tabular}{|c|c|c|c|c|}
\hline Input data & Filter step-out & No. of samples & Filter type & Output Data \\
\hline Seismic reflection & $(1,1,1)$ & $\begin{array}{c}\text { Three (One each in the inline, } \\
\text { crossline and time directions) }\end{array}$ & Median & Raw dip \\
\hline Raw dip and azimuth & $(0,0,5)$ & Five (all in the time direction) & Median & Detailed dip \\
\hline Detailed dip and azimuth & $(5,5,0)$ & $\begin{array}{c}\text { Ten (Five in the inline and five in the } \\
\text { cross line directions) }\end{array}$ & Median & Background dip \\
\hline
\end{tabular}

\subsection{Computing Volumetric Dip and Azimuth Data}

A pre-requisite for computing multi-trace seismic attributes is the knowledge of the dip of the seismic events in the volume [13]. The seismic dip is computed with reference to the $3 \mathrm{D}$ survey grid in the inline, crossline and temporal axes. The data is then stored as inline and crossline dip, equivalent to polar dip and azimuth respectively [12]. The first dip volume was calculated directly from the seismic data using a fast steering filter algorithm based on the analysis of the vertical and horizontal gradient of the amplitude data. Three samples, one each in the inline, crossline and time directions were used for the computation (i.e. step-out is 1, 1, 1). The filtering distance is $75 \mathrm{~m}$ in the inline direction (Inline spacing of $25 \mathrm{~m} \times 3$ samples) and $37.5 \mathrm{~m}$ in the crossline direction (Crossline spacing of $12.5 \mathrm{~m} \times 3$ samples). In the time $(\mathrm{Z})$ direction, the filtering length is $12 \mathrm{msec}$ (sampling rate of $4 \mathrm{msec} \times 3$ samples). This data is referred to as the "raw dip and azimuth volume". The second dip data was computed by applying edge preserving median filter to the raw dip and azimuth volume in the temporal (vertical) direction to attenuate localized noise along structural dips while highlighting subtle structural features. Five samples were used in the temporal direction and the filtering distance is $20 \mathrm{msec}$ (sampling rate of $4 \mathrm{msec} \times 5$ samples). This data contain localized dip and azimuth of the traces and is referred to as the "detailed dip and azimuth volume". The third dip data was computed by applying edge preserving smoothing filter to the detailed dip and azimuth volume in the inline and crossline directions to smoothen localized noise using ten samples along a filtering distance of $250 \mathrm{~m}$ in the inline direction (Inline spacing is $25 \mathrm{~m} \mathrm{x} 10$ samples) and $125 \mathrm{~m}$ in the crossline direction (Crossline spacing is $12.5 \mathrm{~m} \times 10$ samples). No filtering was applied in the temporal (vertical) direction. This data is the "background dip and azimuth volume". This volume is highly smoothened to highlight broad structural trends. The dip data are displayed in a gradational grey-white scale with positive dips represented in white and negative dips in black. The transition from dark to light shades represents moderately dipping events. The parameter setting applied to computing the data are summarized in Table 1 . Comprehensive description of the mathematics of computing dip and azimuth volumes applied to the data can be found in [5].

\subsection{Computing Volumetric Seismic Maximum Curvature Data}

An attribute that detects and highlights the maximum curvature of neighboring trace pairs was computed to highlight structural trend. In 2D, a curve in the xy-plane can be thought of as a consecutive string of arcs of a circle characterized by variable centers and radii. The curvature, $k$, at any given point on this curve is the reciprocal of the radius, $\mathrm{R}$, of the particular arc segment at that point (Fig. 4a). A sense for both the sign and magnitude of curvature of any curve can be obtained by replacing the radii by vectors normal to the curve. The configuration of normal vectors on the curve illustrates the pattern of curvature such that planar surfaces have zero curvature, anticlines have positive curvature and synclines display negative curvature.

In $3 \mathrm{D}$, at any point $P$ on a curved surface, there exists many ways of slicing along one of many section planes with orientations such that they contain the surface normal $\mathrm{n}$ at point $P$ (Fig. $4 \mathrm{~b}$ ). The trace of the surface is usually curved and is called the normal curvature of the surface $\left(\mathrm{k}_{n}\right)$. The 
magnitude of the normal curvature $\mathrm{k}_{n}$ is dependent on the chosen normal section and a change in the magnitude of normal curvature is obtained if the section plane is progressively turned about the surface normal $n$. Curvature in $3 \mathrm{D}$ represents the values of the radii of the two orthogonal circles fit tangent to a surface. Since curvature is the reciprocal of the radius of these circles, maximum curvature $\left(\mathrm{k}_{\max }\right)$ represents the circle that fits the tangent to the surface with the largest radius and minimum curvature $\left(\mathrm{k}_{\min }\right)$ is the other circle tangent to the surface with the smallest radius. Curvature values shows you how deformed a surface is, with high values indicating tighter curves (more deformation) and lower values indicating less curve (flatter events).

\subsection{Computing Multitrace Seismic Maximum Curvature using Dip and Azimuth Data}

Seismic curvature is largely dependent on the dip of the seismic traces [5]. By applying dip-steering techniques, curvature computation is along trace-to-trace guided by the local dip and azimuth at every position along the track (Fig. 5a). However, the trace segments are aligned horizontally without the application of dip-steering (Fig. 5b) thereby very likely to provide erroneous curvature estimates of seismic reflection events in the presence of seismic dip.

The input data for computing multi-trace maximum curvature are the seismic reflection and detailed dip volumes. The latter is used because dip and azimuth variations due to discontinuities are preserved. Therefore, flexures and subtle structural features are highlighted [14]. A time gate of $+24 \mathrm{~ms}$ and $-24 \mathrm{~ms}$, equivalent to the average seismic wavelength within the window of investigation, and a step-out of 1,1 (i.e. two samples), one sample each in the inline and crossline directions were used in computing the attribute. This implies that seismic maximum curvature was computed along every inline and crossline steered by detailed dip data. The attribute was not computed in the temporal (vertical direction) to minimize the effects of artefacts that can potentially mask structural features of interest along time slices (Marfurt, personal communication). The data are displayed in blue-white-red colour display to represent negative, zero and positive maximum curvature respectively. All trace pairs defined by the Inline, crossline and time position were computed using the full-block extension. The parameter setting for multi-trace maximum curvature computation is presented in Table 2.

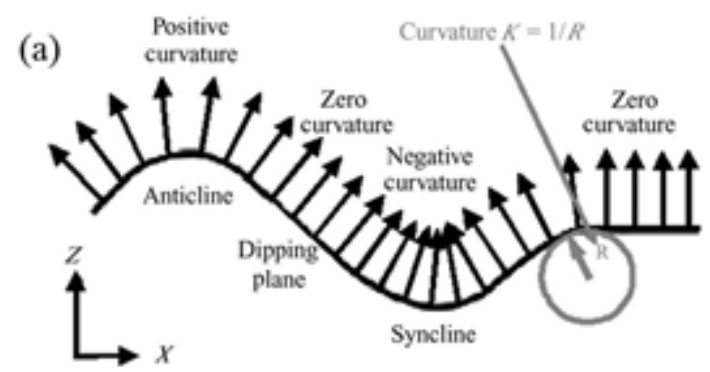

(b)

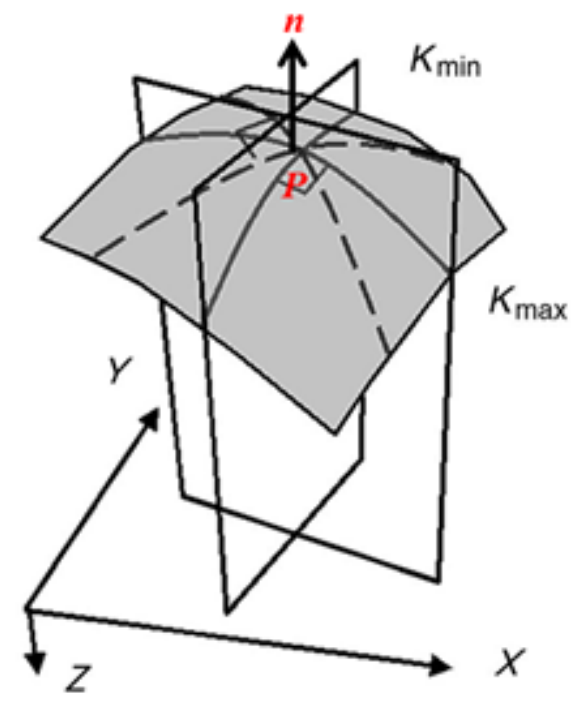

Figure 4. Schematic illustration of the curvature of a point $P$ on a curve and a quadratic surface with a surface normal $n$ at the point (Roberts, 2001).

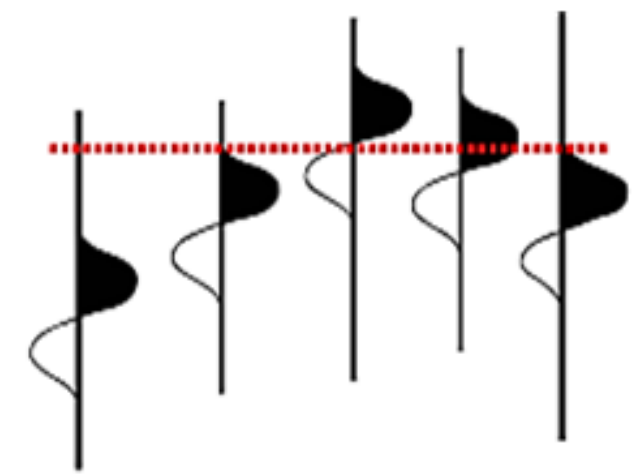

(b)

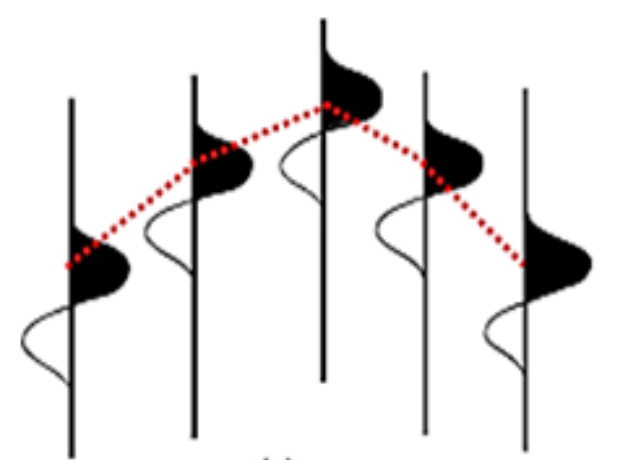

(a)

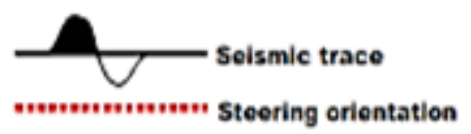

Figure 5. Cross sectional schematic illustration of dip-steering computation techniques applied to the data. 
Table 2. Parameter setting for multitrace maximum curvature computation

\begin{tabular}{|c|c|c|c|c|c|c|}
\hline Input data & $\begin{array}{c}\text { Time gate } \\
(\mathrm{msec})\end{array}$ & Extension & $\begin{array}{c}\text { Trace } \\
\text { step-out }\end{array}$ & No. of samples & Dip-steering & Output data \\
\hline $\begin{array}{c}\text { Seismic reflection } \\
\text { and detailed dip } \\
\text { and azimuth }\end{array}$ & $(-24,24)$ & Full block & $(1,1)$ & $\begin{array}{c}\text { One each in the } \\
\text { inline and } \\
\text { crossline } \\
\text { directions }\end{array}$ & Full & $\begin{array}{c}\text { Maximum } \\
\text { curvature }\end{array}$ \\
\hline
\end{tabular}
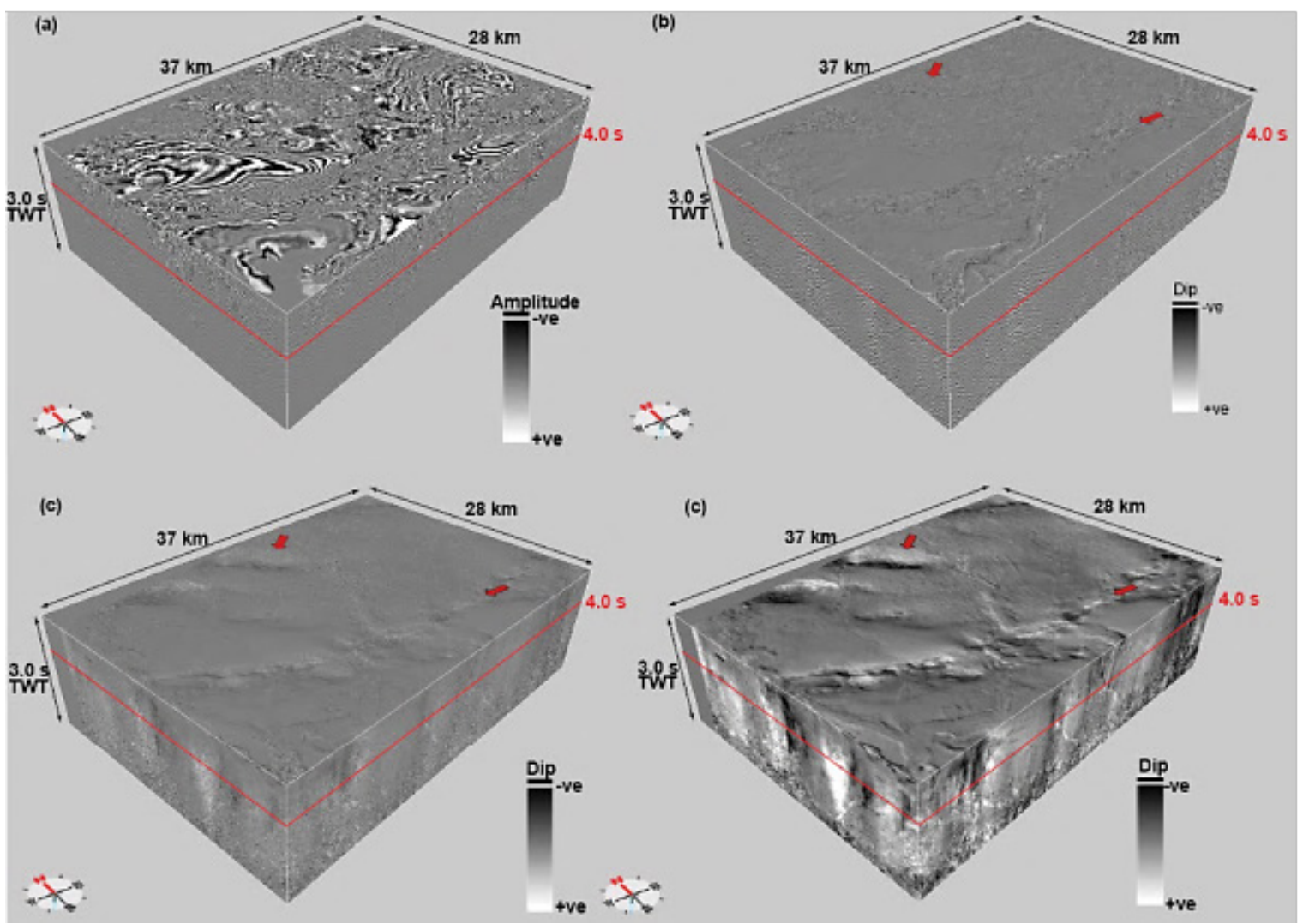

(c)
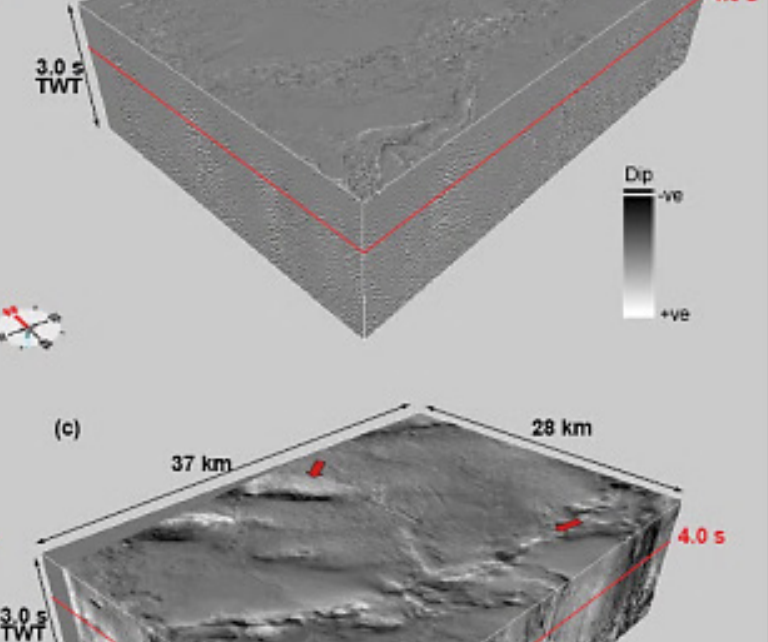

3
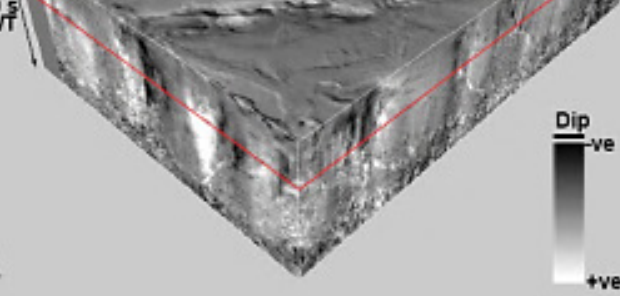

Figure 6. Perspective views of seismic reflection volume (a) raw dip and azimuth volume (b), detailed dip and azimuth volume (c) and background dip and azimuth volume (d) spanning $3.0 \mathrm{~s}$ to $6.0 \mathrm{sec}$ two way travel time. The red outline indicates the location of time slices extracted at $4.0 \mathrm{sec}$ from the data and shown in Figure 5. Vertical exaggeration is $\sim \mathrm{x} 3$. 

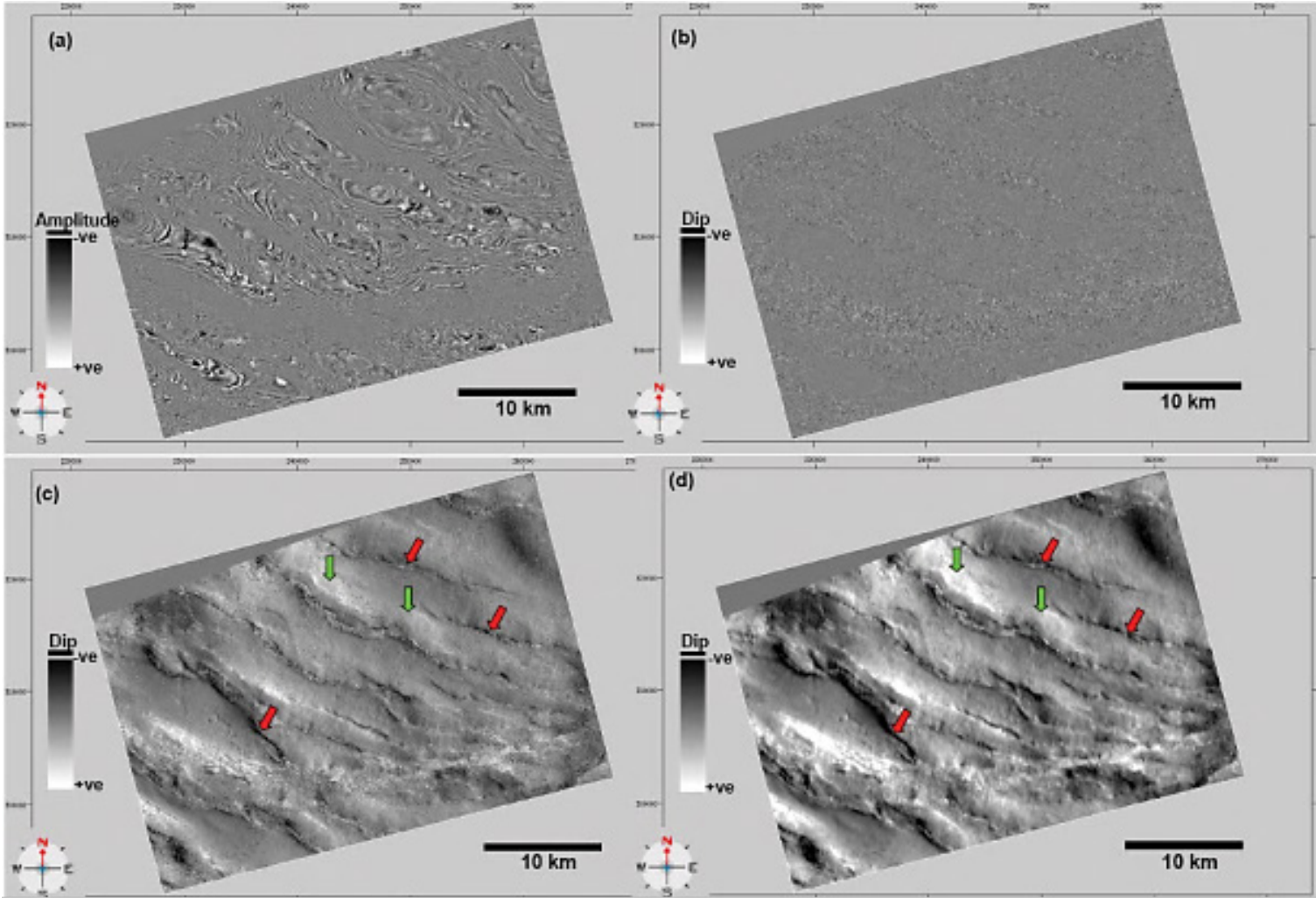

Figure 7. Time slices extracted at $4.0 \mathrm{sec}$ two way travel time from seismic reflection volume (a) raw dip and azimuth volume (b) detailed dip and azimuth volume (c) and background dip and azimuth volume (d).

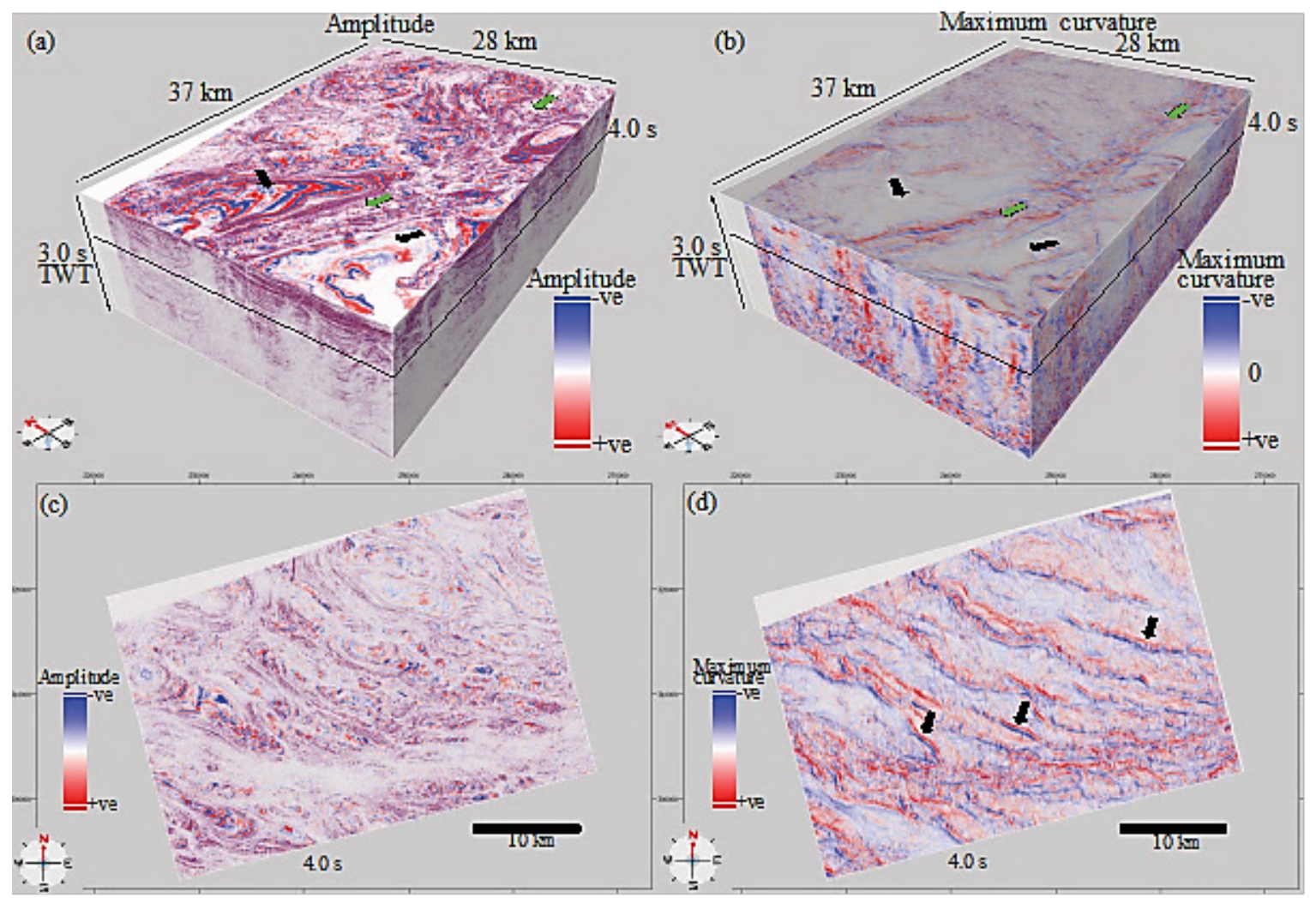

Figure 8. Perspective views of seismic reflection volume (a) and maximum curvature volume (b) spanning $3.0 \mathrm{~s}$ to $6.0 \mathrm{sec}$ two way travel time. The black outline shows the location of time slices extracted at $4.0 \mathrm{sec}$ from the data. Vertical exaggeration is $\sim \mathrm{x} 3$. Figures $7 \mathrm{c}$ and d are timeslices extracted at $4.0 \mathrm{sec}$ two way travel time from the seismic reflection and maximum curvature respectively. 


\section{Results and Discussion}

Figure 6 shows the input seismic reflection volume and three structurally improved dip volumes spanning $3.0 \mathrm{~s}$ to 6.0 sec two-way travel time. The improved contrast of dipping anomalies becomes apparent as additional filtering is applied to the raw dip data calculated directly from the seismic reflection data (Figs. 6b, 6c and 6d). The red arrows in Figure 6 highlight major zones of dip anomalies hardly recognizable in the seismic reflection volume (Fig. 6a). Figure 7 is a time slice display of the attributes extracted from the data volumes at $4.0 \mathrm{sec}$ two-way travel time. The extreme ends of the bar (dark and light shades) represent zones of high dipping anomalies in the data. The red arrows highlight a clearly defined WNW-ESE trend and discontinuities occur at the location of high negative dips while the light shades (green arrows) represent zones of high positive structural dips representing folded data adjacent to the discontinuities. Figure 8 shows seismic amplitude and maximum curvature data volumes spanning $3.0 \mathrm{sec}$ and $6.0 \mathrm{sec}$ two-way travel times. The green arrows highlight zones of intense maximum curvature trending NW-SE, while the black arrow highlight zones of weak curvature. Comparing the two attribute data volumes, there is a good correlation between the pattern of reflectivity and curvature. The black arrows show a good correlation between strong and horizontal pattern of reflectivity with flatter curvature, implying that the latter represent a zone of little or no deformation. The green arrows indicate weak pattern of reflectivity correlating with an arcuate NW-SE trending zone of anomalously high maximum curvature, implying that curvature is likely due to the deformation of the data in the area. Figure 8 shows seismic reflection and maximum curvature time slices extracted from the data volumes at $4.0 \mathrm{sec}$ two-way travel time. In the latter, a clearly defined pattern of WNW-ESE trending zone of curvature characterized by the juxtaposition of positive and negative maximum curvature anomalies is highlighted (black arrows). The orientation and position of the displaced fault blocks can potentially be easily predicted and the structural pattern elucidated. The separation between the curvature anomalies (positive and negative) has in the past been used as a qualitative means of predicting displacements in dip-slip faults [6]. In addition, seismic curvature attribute is very sensitive to brittle deformation and high values of curvature can in most cases be due to intense brittle deformation [13]. In this case study, negative maximum curvature (blue) appears to be behind positive curvature (red), implying that the folded hanging wall block is above the footwall block. This structural pattern is typical of thrust tectonics in the Niger Delta compressional structural domain. This pattern of curvature anomaly provides the interpreter with a rapid reconnaissance tool for understanding the structural pattern of an area prior to detailed interpretation.

\section{Conclusions}

A measure of normal curvature that highlights the largest absolute curvature of seismic reflectors was calculated along structural dips. Time slices extracted from the seismic attribute data has revealed a well-defined WNW-ESE trending discrete zones of dip and curvature anomalies with enhanced contrast not clearly seen in the seismic reflectivity data alone. The structures are interpreted to represent thrust faults and folds formed by gravity-induced compressional deformation in the deep-water Niger Delta. The improved geometrical images of the discontinuities can facilitate a faster and more reliable manual interpretation of faults and provide reliable inputs for calculating reliable subsurface structural models of the area.

\section{Acknowledgements}

The authors are grateful to Petroleum Geo-Services (UK) for providing the seismic data and permission to use the data. dGB Earth Sciences is thanked for donating OpendTect seismic interpretation software for academic use.

\section{REFERENCES}

[1] K.J. Marfurt, V. Sudakher, A. Gerszenkorn, K.D. Crawford, and S.E. Nissen. Coherency calculations in the presence of structural dips, Geophysics, Vol. 64, 104-111, 1999.

[2] S. Al-Dossary, K.J. Marfurt. 3D volumetric multi-spectral estimates of reflector curvature and rotation, Geophysics, Vol. 17, 41-45, 2006.

[3] S. Chopra, K.J., Marfurt. Coherence and curvature attributes on pre-conditioned seismic data, The Leading Edge, Vol. 30, 386-393, 2011.

[4] M.S. Bahorich, S.L., Farmer. 3-D seismic coherency for faults and stratigraphic features, The Leading Edge, Vol. 14, 1053-1058, 1995.

[5] K.M. Tingdahl, P. de Groot. Post-stack dip and azimuth processing, Journal of Seismic Exploration, Vol. 12, 113-116, 2003.

[6] P. Klein, L. Richard, H. James. 3D curvature attributes: a new approach for seismic interpretation, First Break, 26, 105-111, 2008.

[7] R.J. Lisle. Detection of zones of abnormal strains in structures using Gaussian curvature analysis, AAPG Bulletin, Vol. 78, 1811-1818, 1994.

[8] A. Roberts. Curvature attributes and their application to 3D interpreted horizons, First Break, 19, 85-99, 2001.

[9] B. Jibrin. Enhanced detection of faults using dip-steered multitrace similarity computation techniques: Example using offshore Niger Delta 3D seismic data, The Leading Edge, Vol. 33, 428-434, 2014.

[10] R.J. Knipe, G. Jones, and Q.J. Fisher. Faulting, fault seal and 
fluid flow in hydrocarbon reservoirs: an introduction, Geological society of London Special Publication, 147, VII-XXI, 1998

[11] R. Sorkhabi, Y. Tsuji. The place of faults in petroleum traps, in Sorkhabi, R. and Y. Tsuji, (Ed), Faults, fluid flow and petroleum traps, American Association of Petroleum Geologists Memoir, Vol. 85, 1-31, 2005.

[12] P.R. Cobbold, B.J. Clark, H. Loseth. Structural consequences of fluid overpressures and seepage forces in the outer thrust belt of the Niger Delta, Petroleum Geoscience, Vol. 15, No. 1, 3-15, 2009.

[13] F. Brouwer, A. Huck. An integrated workflow to optimize discontinuity attributes for imaging of faults: Presented at 31st Annual Conference of GCSSEPM, Attributes: New views on seismic imaging - their use in exploration and production, 2011.

[14] F. Brouwer. Creating a good steering cube, Opendtect manual (www.opendtect.org). 